\title{
Análise de agrupamento na seleção de modelos de regressão não-lineares para curvas de crescimento de ovinos cruzados
}

\author{
Cluster analysis applied to nonlinear regression models selection to growth curves of crossed lambs
}

\author{
Fernanda Gomes da Silveira $^{\mathrm{I}^{*}}$ Fabyano Fonseca e Silva $^{\mathrm{I}}$ Paulo Luiz Souza Carneiro ${ }^{\mathrm{II}}$ \\ Carlos Henrique Mendes Malhado ${ }^{\text {II }}$ Joel Augusto Muniz ${ }^{\text {II }}$
}

\section{RESUMO}

\begin{abstract}
Este estudo teve como objetivo utilizar a análise de agrupamento para classificar modelos de regressão não-lineares usados para descrever a curva de crescimento de ovinos cruzados, tendo em vista os resultados de diferentes avaliadores de qualidade de ajuste. Para tanto, utilizaram-se dados de pesoidade dos seguintes cruzamentos entre raças de ovinos de corte: Dorper x Morada Nova, Dorper x Rabo Largo e Dorper x Santa Inês. Após a indicação do melhor modelo, objetivou-se ainda aplicar a técnica de identidade de modelos a fim de identificar o cruzamento mais produtivo. Foram ajustados doze modelos não-lineares, cuja qualidade de ajuste foi medida pelo coeficiente de determinação ajustado, critérios de informação de Akaike e Bayesiano, erro quadrático médio de predição e coeficiente de determinação de predição. A análise de agrupamento indicou o modelo Richards como o mais adequado para descrever as curvas de crescimento dos três grupos genéticos considerados, $e$ os testes de identidade de modelos indicaram o cruzamento Dorper x Santa Inês como sendo o mais indicado para a pecuária local.
\end{abstract}

Palavras-chave: classificação multivariada, identidade de modelos, Ovis aries.

\section{ABSTRACT}

This study had the objectives to use the cluster analysis in order to classify nonlinear regression models used to describe the growth curve in relation to different quality fit evaluators. Were utilized weight-age data from the following crossbred beef lambs Dorper x Morada Nova, Dorper x Rabo Largo e Dorper x Santa Inês. After the choice of the best model, we aimed also to apply the model identity in order to identify the most efficient crossbred group. Eleven nonlinear models were used, whose fit quality was measured by determination coefficient, Akaike information criterion, Bayesian information criterion, mean quadratic error of prediction and predicted determination coefficient. The cluster analysis indicated the Richards as the best model for the three data sets, and the model identity tests revealed that the Dorper x Santa Inês crossbred group was recommend for meat production.

Key words: multivariate classification, model identity, Ovis aries.

\section{INTRODUÇÃO}

Em geral, raças naturalizadas ou locais de ovinos de corte do Nordeste brasileiro são adaptadas às condições de solo e clima dessa região, conseguindo assim um bom desempenho de crescimento em condições quase sempre limitantes à criação de outras raças. Segundo MALHADO et al. (2009), uma das formas de aumentar a produção de carne nessa região é a utilização de cruzamentos de raças locais com raças exóticas especializadas para a produção de carne, como, por exemplo, a raça Dorper. De acordo com CARNEIRO et al. (2007), o sucesso de tais cruzamentos pode ser avaliado de forma efetiva por meio da análise das curvas de crescimento dos animais, as quais relacionam o peso com intervalos pré-definidos de tempo.

Modelos de regressão não-linear têm se mostrado adequados para descrever estas curvas, pois

'Departamento de Estatística, Universidade Federal de Viçosa (UFV), 36570-000, Viçosa, MG, Brasil. E-mail: fernandaphn@yahoo.com.br.*Autor para correspondência

"Departamento de Ciências Biológicas, Universidade Estadual do Sudoeste da Bahia (UESB), Jequié, BA, Brasil.

IIIDepartamento de Ciências Exatas, Universidade Federal de Lavras (UFLA), Lavras, MG, Brasil. 
apresentam parâmetros que podem ser interpretados biologicamente, como o peso adulto e a taxa de maturidade (MAZZINI et al., 2005). Porém, a escolha dos melhores modelos não tem sido tarefa fácil, uma vez que diferentes avaliadores de qualidade de ajuste são propostos na literatura, e cada um preconiza determinada característica, como, por exemplo, a minimização dos erros de ajustes, a habilidade de predição e a simplicidade do modelo(SILVEIRA, 2010). Tal escolha é ainda mais difícil quando se tem diferentes populações, ou cruzamentos, pois muitas vezes o modelo que melhor representou o crescimento dos animais de uma população pode não ser o melhor para as demais.

Além da escolha do melhor modelo, ao se considerar várias populações, o pesquisador também tem interesse em comparar os parâmetros das curvas a fim de indicar para qual das populações o processo de crescimento foi mais eficiente. Segundo REGAZZI (2003), a técnica de identidade de modelos é a mais indicada para tal finalidade, porém, para aplicá-la, um mesmo modelo de regressão deve ser ajustado para todas as populações, o que implica a necessidade de utilização de métodos que permitam indicar quais modelos são plausíveis para todas elas.

A análise de agrupamento, a qual possibilita a formação de grupos homogêneos de acordo com a avaliação simultânea de várias características de interesse, pode ser útil para agrupar modelos cujos resultados dos avaliadores de qualidade de ajuste sejam similares dentro de cada população. Assim, os modelos comuns aos grupos representativos dos melhores ajustes em cada população podem ser identificados por meio de medidas de interseção (SILVEIRA, 2010).

De acordo com os aspectos apresentados, o objetivo principal do presente trabalho foi utilizar a análise de agrupamento para classificar modelos de regressão não-lineares, usados para descrever a curva de crescimento, tendo em vista os resultados de diferentes avaliadores de qualidade de ajuste ao se considerar dados de peso-idade de três diferentes cruzamentos de ovinos de corte. Após a escolha do modelo mais adequado, objetivou-se também aplicar a técnica de identidade de modelos para de identificar o cruzamento mais produtivo.

\section{MATERIAL E MÉTODOS}

Os dados utilizados neste estudo são oriundos de um experimento que foi conduzido na Estação Experimental de Jaguaquara, pertencente à Empresa Baiana de Desenvolvimento Agrícola S.A., localizada no Município de Jaguaquara, BA, microrregião administrativa de Jequié, BA, de 2003 a 2005.
Foram avaliados os produtos $\mathrm{F} 1$, compreendendo 156 borregos, dos cruzamentos Dorper x Morada Nova (DMN); Dorper x Rabo Largo (DRL) e Dorper x Santa Inês (DSI). Destes, 36 DMN (26 machos e 10 fềmeas), 61 DRL (36 machos e 25 fêmeas) e 59 DSI (31 machos e 28 fêmeas) são provenientes do cruzamento de 69 matrizes pluríparas (23 Santa Inês, 23 Morada Nova e 23 Rabo Largo) com 2 reprodutores da raça Dorper. Os animais foram pesados a partir dos 20 dias de idade até os 300 dias de idade em intervalos de aproximadamente 15 dias.

Após o desmame, que ocorreu aos 84 dias, os produtos $\mathrm{F} 1$ foram mantidos em sistema de produção semi-intensivo, com pastejo durante o dia em áreas cultivadas com capim Panicum maximum e Brachiaria decumbens com sal mineral à disposição, e recolhidos no final da tarde para o aprisco. Durante o inverno, período mais seco do ano (junho a outubro), os animais receberam suplementação com mistura múltipla comercial.

Os modelos de regressão não-linear apresentados na tabela 1 foram ajustados aos dados médios de peso-idade de ovinos por meio do PROC MODEL do SAS $^{\circledR}$ (SAS INSTITUTE, 2002) via método dos quadrados mínimos ordinários com algoritmo de Gauss-Newton. Para todos os modelos apresentados na tabela 1 (SILVEIRA, 2010), o parâmetro $\beta_{1}$ representa o peso adulto do animal; o $\beta_{2}$ não apresenta interpretação prática; e o parâmetro $\beta_{3}$ a taxa de maturidade. Os modelos que apresentam o parâmetro $\beta_{4}$ possuem ponto de inflexão variável, e os demais modelos ou apresentam o ponto de inflexão fixo, ou não o possuem, comoé o caso dos modelos Brody e Michaelis-Menten. Para todos os modelos, assumiu-se que $\varepsilon_{i} \sim N\left(0, \sigma^{2}\right)$.

Foram utilizados cinco avaliadores da qualidade de ajuste: coeficiente de determinação ajustado $\left(\mathrm{R}_{\text {aj }}^{2}\right.$ ), critério de informação de Akaike (AIC), critério de informação bayesiano (BIC), erro quadrático médio de predição (MEP) e coeficiente de determinação de predição $\left(\mathrm{R}_{\mathrm{p}}^{2}\right)$. As expressões desses avaliadores também são apresentadas de forma detalhada em SILVEIRA(2010).

Após a obtenção dos avaliadores de qualidade para cada modelo, confeccionaram-se então conjuntos de dados multivariados, nos quais os modelos correspondem às unidades, ou aos indivíduos, e os avaliadores às variáveis. Estes foram submetidos à análise de agrupamento no PROC CLUSTER (SAS INSTITUTE, 2002), considerando o método centróide. Nessa análise, obtiveram-se os valores do índice RMSSTD (Root Mean Square Standard Deviation, ou, raiz quadrada do desvio padrão médio), o qual possibilita identificar o número ótimo de cluster de acordo com CECON et al. (2008). Como complemento dessa última análise, utilizou-se o 
Tabela 1 - Modelos de regressão não-linear para descrever curvas de crescimento.

\begin{tabular}{|c|c|c|c|}
\hline Modelo & Função & Modelo & Função \\
\hline A Schnute & $\mathrm{y}_{\mathrm{i}}=\frac{\beta_{1}}{\left(1+\beta_{4} \mathrm{e}^{\left(\beta_{3} \beta_{2}-\mathrm{x}_{\mathrm{i}}\right)}\right)^{\frac{1}{\beta_{4}}}}+\mathrm{e}_{\mathrm{i}}$ & H Meloun II & $y_{i}=\beta_{1}-e^{\left(-\beta_{2}-\beta_{3} x_{i}\right)}+e_{i}$ \\
\hline B Mitscherlich & $\mathrm{y}_{\mathrm{i}}=\beta_{1}\left(1-\mathrm{e}^{\left(\beta_{3} \beta_{2}-\beta_{3} \mathrm{x}_{\mathrm{i}}\right)}\right)+\mathrm{e}_{\mathrm{i}}$ & N Brody & $y_{i}=\beta_{1}\left(1-\beta_{2} e^{-\beta_{3} x_{i}}\right)+e_{i}$ \\
\hline C Richards & $\mathrm{y}_{\mathrm{i}}=\frac{\beta_{1}}{\left(1+\mathrm{e}^{\left(\beta_{2}-\beta_{3} \mathrm{x}_{\mathrm{i}}\right)}\right)^{\frac{1}{\beta_{4}}}}+\mathrm{e}_{\mathrm{i}}$ & O von Bertalanffy & $\mathrm{y}_{\mathrm{i}}=\beta_{1}\left(1-\beta_{2} \mathrm{e}^{-\beta_{3} \mathrm{x}_{\mathrm{i}}}\right)^{3}+\mathrm{e}_{\mathrm{i}}$ \\
\hline D Gompertz & $\mathrm{y}_{\mathrm{i}}=\beta_{1} \mathrm{e}^{\left(-\mathrm{e}\left(\beta_{2}-\beta_{3} \mathrm{x}_{\mathrm{i}}\right)\right)}+\mathrm{e}_{\mathrm{i}}$ & P Michaelis-Menten & $\mathrm{y}_{\mathrm{i}}=\frac{\beta_{1} \mathrm{x}_{\mathrm{i}}}{\mathrm{x}_{\mathrm{i}}+\beta_{2}}+\mathrm{e}_{\mathrm{i}}$ \\
\hline E Logístico & $\mathrm{y}_{\mathrm{i}}=\frac{\beta_{1}}{\left(1+\mathrm{e}^{\left(\beta_{2}-\beta_{3} \mathrm{x}_{\mathrm{i}}\right)}\right)}+\mathrm{e}_{\mathrm{i}}$ & $\begin{array}{l}\text { Q Michaelis-Menten } \\
\text { Modificado }\end{array}$ & $\mathrm{y}_{\mathrm{i}}=\frac{\beta_{2} \beta_{3}^{\beta_{4}}+\beta_{1} \mathrm{x}_{\mathrm{i}}^{\beta_{4}}}{\beta_{3}^{\beta_{4}}+\mathrm{x}_{\mathrm{i}}^{\beta_{4}}}+\mathrm{e}_{\mathrm{i}}$ \\
\hline F Meloun I & $\mathrm{y}_{\mathrm{i}}=\beta_{1}-\beta_{2} \mathrm{e}^{\left(-\beta_{3} \mathrm{x}_{\mathrm{i}}\right)}+\mathrm{e}_{\mathrm{i}}$ & & \\
\hline
\end{tabular}

PROC TREE (SAS INSTITUTE, 2002), que fornece o dendrograma e permite verificar quais modelos pertenciam aos diferentes grupos obtidos pela discriminação estatística em relação aos avaliadores de qualidade.

Após a escolha de um modelo que se ajustou bem aos três grupos genéticos, aplicou-se o teste de identidade de modelos proposto por REGAZZI (2003) com o intuito de verificar diferenças nas estimativas dos parâmetros entre os três cruzamentos avaliados. Para tanto, utilizou-se o PROC MODEL (SAS INSTITUTE, 2002) com auxílio de variáveis dummy.

\section{RESULTADOS E DISCUSSÃO}

Nas tabelas 2, 3, e 4, são apresentados, respectivamente para os cruzamentos DMN, DRL e DSI, os resultados dos avaliadores da qualidade de ajuste para cada modelo. Nota-se, nas tabelas 2, 3, e 4, que, em média, os ajustes dos modelos para o grupo DSI (Tabela 4) apresentaram maior qualidade que os demais grupos, uma vez que foram observados os maiores valores para o $\mathrm{R}_{\text {aj }}{ }_{\mathrm{j}}$ e $\mathrm{R}_{\mathrm{p}}^{2}$ e os menores para AIC, BIC e MEP. Essas informações indicam que os dados médios do grupo DSI apresentam um comportamento mais característico de curva decrescimento(Figura 1), uma vez que os modelos específicos para descrever este fenômeno se ajustaram melhor.
Em relação à variabilidade relativa dos avaliadores, nota-se que o MEP é o que apresenta maior $\mathrm{CV}$, talvez porque esse avaliador, calculado com base na remoção sucessiva de observações dos conjuntos de dados originais, represente a capacidade dos modelos em se ajustarem bem na presença de dados faltantes e, comojá demonstrado por NOBRE et al. (1987), a qualidade desses modelos varia significativamente com a perda de observações. Quanto às medidas baseadas no conceito de coeficiente de determinação $\left(\mathrm{R}_{\mathrm{aj}}^{2} \mathrm{e} \mathrm{R}_{\mathrm{p}}^{2}\right)$, foram aquelas que apresentaram menores $\mathrm{CV}^{\prime} \mathrm{s}$, indicando que este avaliador realmente apresenta baixa variabilidade. Quanto a essa informação, OLIVEIRA et al. (2000) relatam que as diferenças entre os $\mathrm{R}_{\text {aj }}^{2}$ de diferentes modelos usados no estudo de curvas de crescimento geralmente são irrisórias, e recomendam outros avaliadores.

Para todos os grupos genéticos avaliados, o número ótimo de cluster encontrado foi três, ou seja, os onze modelos considerados foram condensados em apenas três grupos distintos. Na tabela 5, são apresentados os clusters, ordenados pelos valores mais plausíveis para os avaliadores da qualidade de ajuste, com os seus respectivos modelos e médias de tais avaliadores. Ao verificar quais modelos são pertencentes aos clusters 1, os quais contêm os melhores modelos, nota-se que o modelo C (Richards) encontra-se entre os 
Tabela 2 - Resultados dos avaliadores da qualidade de ajuste para os modelos do grupo genético Dorper x Morada Nova (DMN) ${ }^{(1)}$.

\begin{tabular}{|c|c|c|c|c|c|}
\hline \multirow{2}{*}{ Modelo } & \multicolumn{5}{|c|}{ - } \\
\hline & $\mathrm{R}_{\text {aj }}^{2}$ & AIC & $\mathrm{BIC}$ & MEP & $\mathrm{R}_{\mathrm{p}}^{2}$ \\
\hline A - Schnute & 0,9503 & 56,6926 & 58,8167 & 4,9633 & 0,9922 \\
\hline B - Mitscherlich & 0,9938 & 25,4254 & 27,5495 & 0,3664 & 0,9994 \\
\hline C - Richards & 0,9904 & 34,073 & 36,9052 & 0,6186 & 0,9990 \\
\hline D - Gompertz & 0,9904 & 32,073 & 34,1971 & 0,6186 & 0,9990 \\
\hline E - Logístico & 0,9859 & 37,799 & 39,9231 & 0,9165 & 0,9985 \\
\hline F - Meloun I & 0,9938 & 25,4254 & 27,5495 & 0,3664 & 0,9994 \\
\hline H - Meloun II & 0,9938 & 25,4254 & 27,5495 & 0,3664 & 0,9994 \\
\hline $\mathrm{N}$ - Brody & 0,9938 & 25,4254 & 27,5495 & 0,3665 & 0,9994 \\
\hline O - von Bertalanffy & 0,9917 & 29,8682 & 31,9923 & 0,5262 & 0,9991 \\
\hline P - Michaelis-Menten & 0,9873 & 35,3572 & 36,7733 & 0,6887 & 0,9989 \\
\hline Q - Michaelis-Menten Mod. & 0,9956 & 24,5512 & 27,3834 & 0,2982 & 0,9995 \\
\hline Média & 0,9879 & 32,0105 & 34,1990 & 0,9179 & 0,9986 \\
\hline Desvio-padrão & 0,0128 & 9,4438 & 9,3858 & 1,3547 & 0,0021 \\
\hline $\mathrm{CV} \%$ & 1,2977 & 29,5022 & 27,4446 & 147,5894 & 0,2122 \\
\hline
\end{tabular}

(1) Coeficiente de determinação ajustado $\left(\mathrm{R}^{2}{ }_{\mathrm{aj}}\right)$, critério de informação de Akaike (AIC), critério de informação bayesiano (BIC), erro quadrático médio de predição (MEP), coeficiente de determinação de predição $\left(\mathrm{R}_{\mathrm{p}}^{2}\right)$ e coeficiente de variação $(\mathrm{CV} \%)$.

melhores para descrever o crescimento dos grupos DRL e DSI, enquanto que o modelo Q (Michaelis-Menten Modificado) encontra-se entre os melhores para descrever o crescimento dos grupos DMN e DSI. Entretanto, optou-se pelo modelo $\mathrm{C}$, devido à maior facilidade de interpretação de seus parâmetros, e também pelo fato de tal modelo ser mais utilizado para descrição de curvas de crescimento de ovinos (LOBO et al., 2006) em relação ao modelo Q.
De acordo com esses testes de identidade de modelos (Tabela 6 ), nota-se que houve diferenças entre as estimativas do peso adulto $\left(\beta_{1}\right)$ para o cruzamento DRL $\left(\hat{\beta}_{12}=27,4394\right)$ em relação aos cruzamentos DSI $\left(\hat{\beta}_{13}=33,1862\right)$ e $\operatorname{DMN}\left(\hat{\beta}_{11}=33,4791\right)$. Quanto à taxa de maturidade $\left(\beta_{3}\right)$, observa-se que houve diferença significativa para todas as comparações efetuadas, e as estimativas deste parâmetro para os cruzamentos DMN, DRLeDSI foram, respectivamente, $0,0085,0,0275$ e 0,0123.

Tabela 3 - Resultados dos avaliadores da qualidade de ajuste para os modelos do grupo genético Dorper x Rabo Largo (DRL) ${ }^{(1)}$.

\begin{tabular}{|c|c|c|c|c|c|}
\hline \multirow{2}{*}{ Modelo } & \multicolumn{5}{|c|}{ 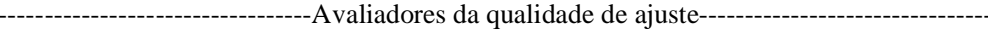 } \\
\hline & $\mathrm{R}_{\text {aj }}^{2}$ & AIC & BIC & MEP & $\mathrm{R}_{\mathrm{p}}^{2}$ \\
\hline A - Schnute & 0,9843 & 47,7172 & 50,2168 & 1,1447 & 0,9985 \\
\hline B - Mitscherlich & 0,9776 & 53,7266 & 56,2262 & 1,3249 & 0,9982 \\
\hline C - Richards & 0,9881 & 43,7096 & 47,0424 & 0,9636 & 0,9987 \\
\hline D - Gompertz & 0,9838 & 48,2012 & 50,7008 & 0,9608 & 0,9987 \\
\hline E - Logístico & 0,9872 & 44,2686 & 46,7682 & 0,7814 & 0,9989 \\
\hline F - Meloun I & 0,9776 & 53,7266 & 56,2262 & 1,3247 & 0,9982 \\
\hline H - Meloun II & 0,9776 & 53,7266 & 56,2262 & 1,3248 & 0,9982 \\
\hline $\mathrm{N}$ - Brody & 0,9776 & 53,7266 & 56,2262 & 1,3251 & 0,9982 \\
\hline O - von Bertalanffy & 0,9821 & 49,8986 & 52,3982 & 1,0561 & 0,9986 \\
\hline P - Michaelis-Menten & 0,9724 & 56,4468 & 58,1132 & 1,5880 & 0,9979 \\
\hline Q - Michaelis-Menten Mod. & 0,9824 & 50,337 & 53,6698 & 1,3900 & 0,9982 \\
\hline Média & 0,9810 & 50,4987 & 53,0741 & 1,1986 & 0,9985 \\
\hline Desvio-padrão & 0,0048 & 4,1877 & 3,9483 & 0,2369 & 0,0003 \\
\hline $\mathrm{CV} \%$ & 0,4899 & 8,2926 & 7,4392 & 19,7631 & 0,0306 \\
\hline
\end{tabular}

\footnotetext{
${ }^{(1)}$ Idem Tabela 2.
} 
Tabela 4 - Resultados dos avaliadores da qualidade de ajuste para os modelos do grupo genético Dorper x Santa Inês (DSI) ${ }^{(1)}$.

\begin{tabular}{|c|c|c|c|c|c|}
\hline \multirow{2}{*}{ Modelo } & \multicolumn{5}{|c|}{ - } \\
\hline & $\mathrm{R}_{\text {aj }}^{2}$ & AIC & BIC & MEP & $\mathrm{R}_{\mathrm{p}}^{2}$ \\
\hline A - Schnute & 0,9520 & 56,0056 & 57,9227 & 3,9977 & 0,9954 \\
\hline B - Mitscherlich & 0,9958 & 21,7798 & 23,6969 & 0,3320 & 0,9996 \\
\hline C - Richards & 0,9959 & 22,1736 & 24,7298 & 0,2628 & 0,9997 \\
\hline D - Gompertz & 0,9963 & 20,1736 & 22,0907 & 0,2603 & 0,9997 \\
\hline E - Logístico & 0,994 & 26,8036 & 28,7207 & 0,4144 & 0,9995 \\
\hline F - Meloun I & 0,9958 & 21,7798 & 23,6969 & 0,3322 & 0,9996 \\
\hline H - Meloun II & 0,9958 & 21,7798 & 23,6969 & 0,3321 & 0,9996 \\
\hline $\mathrm{N}$ - Brody & 0,9958 & 21,7798 & 23,6969 & 0,3322 & 0,9996 \\
\hline O - von Bertalanffy & 0,9965 & 19,2116 & 21,1287 & 0,2479 & 0,9997 \\
\hline P - Michaelis-Menten & 0,9916 & 30,7974 & 32,0755 & 0,5227 & 0,9994 \\
\hline Q - Michaelis-Menten Mod. & 0,9957 & 22,9998 & 25,5560 & 0,3476 & 0,9996 \\
\hline Média & 0,9914 & 25,9349 & 27,9102 & 0,6708 & 0,9992 \\
\hline Desvio-padrão & 0,0131 & 10,4827 & 10,4177 & 1,1049 & 0,0013 \\
\hline CV\% & 1,3249 & 40,4191 & 37,3256 & 164,7121 & 0,1251 \\
\hline
\end{tabular}

${ }^{(1)}$ Idem tabela 2 .

De acordo com resultados da tabela 6, podese inferir que os cruzamentos DMN e DSI apresentam pesos adultos maiores que o do grupo DRL, e que a maior velocidade de crescimento foi constatada para o cruzamento DRL, seguido pelo DSI e DMN. De forma geral, o antagonismo relacionado ao fato de cruzamentos com maiores valores para o peso adulto apresentarem menores valores para a taxa de maturidade pode ser explicado pela correlação negativa entre tais parâmetros, a qual já foi relatada em vários trabalhos (MALHADO et al., 2008; MALHADO et al., 2009). Sob o ponto de vista zootécnico, o cruzamento DSI é o mais indicado para se explorar a produção de carne, pois, se comparado ao DMN, apresentou peso adulto estatisticamente igual e maior taxa de maturidade.

\section{CONCLUSÃO}

A metodologia proposta no presente trabalho mostrou-se adequada para classificar modelos

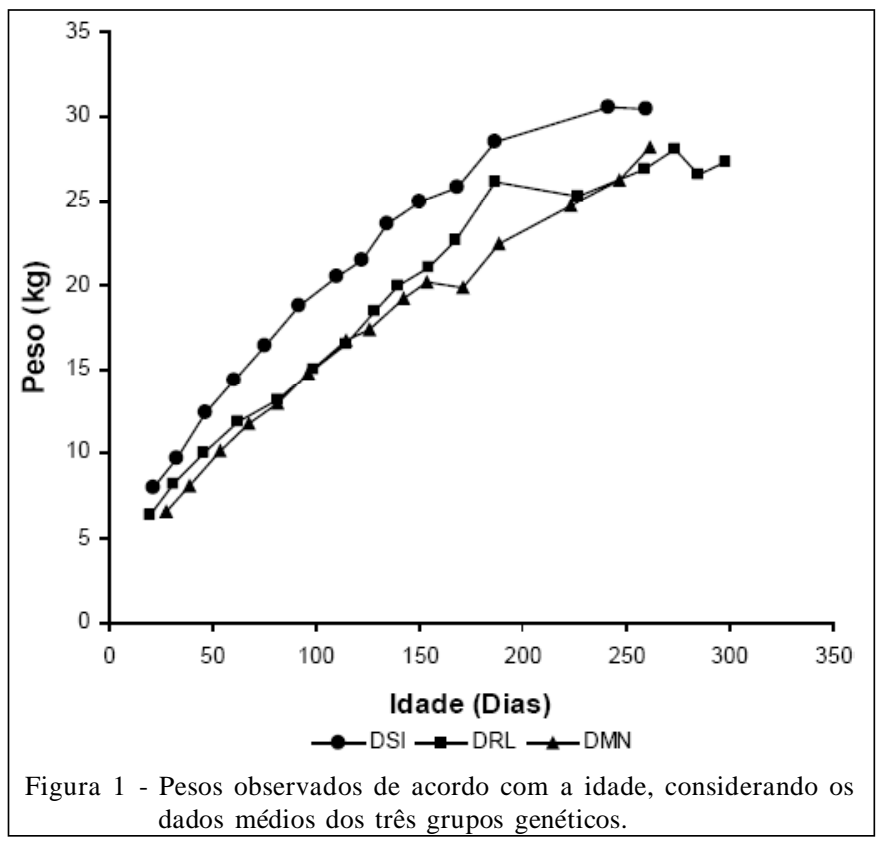

Ciência Rural, v.41, n.4, abr, 2011. 
Tabela 5 - Clusters com os seus respectivos modelos e médias dos avaliadores de qualidade de ajuste (AQ) para cada cruzamento considerado.

\begin{tabular}{|c|c|c|c|c|}
\hline & & Cluster 1 & Cluster 2 & Cluster 3 \\
\hline Cruzam & AQ & Modelos $\{\mathrm{B}, \mathrm{H}, \mathrm{F}, \mathrm{N}, \mathrm{Q}\}$ & Modelos $\{\mathrm{C}, \mathrm{P}, \mathrm{D}, \mathrm{O}, \mathrm{E}\}$ & Modelos $\{\mathrm{A}\}$ \\
\hline \multirow{5}{*}{ DMN } & $\mathrm{R}_{\mathrm{aj}}^{2}$ & 0,9941 & 0,9891 & 0,9503 \\
\hline & AIC & 25,2505 & 33,8340 & 56,6926 \\
\hline & $\mathrm{BIC}$ & 27,5163 & 35,9582 & 58,8167 \\
\hline & MEP & 0,3528 & 0,6737 & 4,9633 \\
\hline & $\mathrm{R}_{\mathrm{p}}^{2}$ & 0,9994 & 0,9989 & 0,9922 \\
\hline \multirow{8}{*}{ DRL } & & Cluster 1 & Cluster 2 & Cluster 3 \\
\hline & AQ & Modelos $\{\mathrm{C}, \mathrm{E}\}$ & Modelos $\{\mathrm{A}, \mathrm{D}, \mathrm{O}, \mathrm{Q}\}$ & Modelos $\{\mathrm{B}, \mathrm{H}, \mathrm{F}, \mathrm{N}, \mathrm{P}\}$ \\
\hline & $\mathrm{R}_{\text {aj }}^{2}$ & 0,9876 & 0,9831 & 0,9765 \\
\hline & AIC & 43,9891 & 49,0385 & 54,2706 \\
\hline & $\mathrm{BIC}$ & 46,9053 & 51,7464 & 56,6036 \\
\hline & MEP & 0,8725 & 1,1379 & 1,3775 \\
\hline & $\mathrm{R}_{\mathrm{p}}^{2}$ & 0,9988 & 0,9985 & 0,9982 \\
\hline & & Cluster 1 & Cluster 2 & Cluster 3 \\
\hline \multirow{6}{*}{ DSI } & AQ & Modelos $\{\mathrm{F}, \mathrm{N}, \mathrm{H}, \mathrm{B}, \mathrm{C}, \mathrm{D}, \mathrm{O}, \mathrm{Q}\}$ & Modelos $\{\mathrm{E}, \mathrm{P}\}$ & Modelos $\{\mathrm{A}\}$ \\
\hline & $\mathrm{R}_{\text {aj }}^{2}$ & 0,9959 & 0,9928 & 0,9520 \\
\hline & AIC & 21,4597 & 28,8005 & 56,0056 \\
\hline & BIC & 23,5366 & 30,3981 & 57,9227 \\
\hline & MEP & 0,30593 & 0,4686 & 3,9938 \\
\hline & $\mathrm{R}_{\mathrm{p}}^{2}$ & 0,9996 & 0,9994 & 0,9954 \\
\hline
\end{tabular}

não-lineares tendo em vista a escolha de um único e melhor modelo para descrever o crescimento dos três grupos genéticos estudados. O modelo Richards foi o que apresentou os melhores ajustes e, de acordo com testes de identidade de modelos, o grupo genético DSI foi o mais recomendado para exploração de carne, uma vez que conciliou valores desejados para peso adulto e taxa de maturidade.

Tabela 6 - Testes de identidade de modelos tendo em vista os parâmetros do modelo Richards que apresentam interpretação biológica ( $\beta_{1 \mathrm{k}} \mathrm{e}$ $\left.\beta_{3 \mathrm{k}}\right)^{(2)}$.

\begin{tabular}{|c|c|c|c|c|c|}
\hline Hipóteses & $\mathrm{n}$ & $\begin{array}{c}\mathrm{SQR}_{\Omega} \\
\text { (Completo) }\end{array}$ & $\begin{array}{c}\mathrm{SQR} \omega \\
\text { (Reduzido) }\end{array}$ & $\chi_{\text {Calculado }}^{2}$ & $\chi_{\text {Tabelado }}^{2}$ \\
\hline $\mathrm{H}_{0}^{(1)}: \beta_{11}=\beta_{12}=\beta_{13}$ vs $\mathrm{H}_{\mathrm{a}}^{(1)}$ & 46 & 15,3649 & 22,5925 & $17,7348^{*}$ & $\chi_{0,05}^{2}(2)=5,9915$ \\
\hline 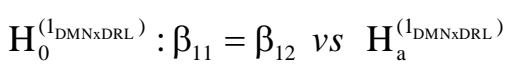 & 32 & 13,1088 & 17,7232 & $9,6509^{*}$ & \\
\hline $\mathrm{H}_{0}^{\left(1_{\mathrm{DMN} \times \mathrm{DSI}}\right)}: \beta_{11}=\beta_{13}$ vs $\mathrm{H}_{\mathrm{a}}^{\left(1_{\mathrm{DMN} \times \mathrm{DSI}}\right)}$ & 29 & 7,2507 & 7,2579 & 0,0287 & $\chi_{0,05}^{2}(1)=3,8415$ \\
\hline $\mathrm{H}_{0}^{\left(1_{\mathrm{DRLXDSI}}\right)}: \beta_{12}=\beta_{13}$ vs $\mathrm{H}_{\mathrm{a}}^{\left(1_{\mathrm{DRLXDSI}}\right)}$ & 31 & 10,3701 & 16,9365 & $15,2068^{*}$ & \\
\hline $\mathrm{H}_{0}^{(3)}: \beta_{31}=\beta_{32}=\beta_{33}$ vs $\mathrm{H}_{\mathrm{a}}^{(3)}$ & 46 & 15,3649 & 20,0642 & $12,2751^{*}$ & $\chi_{0,05}^{2}(2)=5,9915$ \\
\hline $\mathrm{H}_{0}^{\left(3_{\text {DMNxDRL }}\right)}: \beta_{31}=\beta_{32}$ vs $\mathrm{H}_{\mathrm{a}}^{\left(3_{\mathrm{DMN} \times \mathrm{DRL}}\right)}$ & 32 & 13,1088 & 17,7885 & $9,7685^{*}$ & \\
\hline $\mathrm{H}_{0}^{\left(3_{\mathrm{DMN} x \mathrm{DSI}}\right)}: \beta_{31}=\beta_{33}$ vs $\mathrm{H}_{\mathrm{a}}^{\left(3_{\mathrm{DMN} \times \mathrm{DSI}}\right)}$ & 29 & 7,2507 & 8,6619 & $5,1572^{*}$ & $\chi_{0,05}^{2}(1)=3,8415$ \\
\hline $\mathrm{H}_{0}^{\left(3_{\text {DRLXDSI }}\right)}: \beta_{32}=\beta_{33}$ vs $\mathrm{H}_{\mathrm{a}}^{\left(3_{\text {DRLXDSI }}\right)}$ & 31 & 10,3701 & 12,7749 & $6,4652^{*}$ & \\
\hline
\end{tabular}

(2) $\mathrm{O}$ índice $\mathrm{k}$ corresponde aos três grupos genéticos: $\mathrm{k}=1(\mathrm{DMN})$, 2(DRL) e 3(DSI).

* Significativo em nível de 0,05 de probabilidade. 


\section{REFERÊNCIAS}

CARNEIRO, P.L.S. et al. Desenvolvimento ponderal e diversidade fenotípica entre cruzamentos de ovinos Dorper com raças locais. Pesquisa Agropecuária Brasileira, v.42, n.7, p.991-998, 2007. Disponível em: <http://www.scielo.br/ scielo.php?script=sci_arttext \& pid=S $0100-$ 204X2007000700011\&lang=pt>. Acesso em: 13 jul. 2009. doi: 10.1590/S0100-204X2007000700011.

CECON, P.R. et al. Análise de medidas repetidas na avaliação de clones de café 'Conilon'. Pesquisa Agropecuária Brasileira, v.43, n.9, p.1171-1176, 2008. Disponível em: <http://www.scielo.br/ scielo.php?script=sci_arttext\&pid=S0100204X2008000900011\& lang=pt $>$. Acesso em: 17 jul. 2009. doi: 10.1590/S0100204X2008000900011.

LOBO, R.N.B. et al. Parâmetros genéticos de características estimadas da curva de crescimento de ovinos da raça Santa Inês. Revista Brasileira de Zootecnia, v.35, n.3, p.10121019, 2006. Disponível em: <http://www.scielo.br/ scielo.php ? script=sciarttext\&pid=_S 1516 5982006000400011\&lang=pt>. Acesso em: 23 maio, 2009. doi: $10.1590 / \mathrm{S} 1516-35982006000400011$.

MALHADO, C.H.M. et al. Growth curves in Dorper sheep crossed with the local Brazilian breeds, Morada Nova, Rabo Largo, and Santa Inês. Small Ruminant Research, v.84, p.16-21, 2009. Disponível em: <http://www.journals.elsevierhealth.com/ periodicals/rumin/article/PIIS0921448809000807/ abstract?showOmimLinks=true>. Acesso em: 18 out. 2009. doi: 10.1016/j.smallrumres.2009.04.006.

MALHADO, C.H.M. et al. Curva de crescimento em ovinos mestiços Santa Inês x Texel criados no Sudoeste do Estado da
Bahia. Revista Brasileira de Saúde e Produção Animal, v.9, n.2, p.210-218, 2008. Disponível em: <http://revistas.ufba.br/ index.php/rbspa/article/view/1059/595>. Acesso em: 21 fev. 2009.

MAZZINI, A.R. et al. Curva de crescimento de novilhos Hereford: heterocedasticidade e resíduos Autorregressivos. Ciência Rural, v35, n.2, p.422-427, 2005. Disponível em: $<\mathrm{http}: / / \mathrm{www}$.scielo.br/scielo.php? pid=S 0103 $84782005000200028 \&$ script $=$ sciarttext $>$. Acesso em: 15 jan. 2009. doi: $10.1590 / \mathrm{S} 0103-84782005000200028$.

NOBRE, P.R.C. et al. Curvas de crescimento de gado Nelore ajustadas para diferentes freqüências de pesagens. Pesquisa Agropecuária Brasileira, v.22, n 9/10, p.1027-1037, 1987.

OLIVEIRA, H.N. de et al. Comparação de modelos não-lineares para descrever o crescimento de fêmeas da raça Guzerá. Pesquisa Agropecuária Brasileira, v.35, n.9, p.1843-1851, 2000. Disponível em: <http://www.scielo.br/scielo.php?script=sci_arttext \&pid=S0100204X2000000900017\&lang=pt. Acesso em: $11 \mathrm{fev}$. 2009. doi: 10.1590/S0100-204X2000000900017>.

REGAZZI, A.J. Teste para verificar a igualdade de parâmetros e a identidade de modelos de regressão não-linear. Revista Ceres, v.50, n.287, p.9-26, 2003. Disponível em: <http:// www.ceres.ufv.br/CERES/revistas/V50N287P19303.pdf>. Acesso em: 05 out. 2009.

SAS Institute Inc. Statistical Analysis System user's guide. Version 9.0 ed. Cary, 2002. 513p.

SILVEIRA, F.G. da. Classificação multivariada de modelos de crescimento para grupos genéticos de ovinos de corte. 2010. 61f. Dissertação (Mestrado em Estatística Aplicada e Biometria) - Universidade Federal de Viçosa, Viçosa, MG. 